UDC $519.61 ; 519.62 ; 621.372 .82$

DOI: $10.22363 / 2658-4670-2020-28-3-252-273$

\title{
Asymptotic method for constructing a model of adiabatic guided modes of smoothly irregular integrated optical waveguides
}

\author{
Anton L. Sevastianov \\ Peoples' Friendship University of Russia (RUDN University) \\ 6, Miklukho-Maklaya St., Moscow, 117198, Russian Federation
}

(received: June 10, 2020; accepted: September 14, 2020)

The paper considers a class of smoothly irregular integrated optical multilayer waveguides, whose properties determine the characteristic features of guided propagation of monochromatic polarized light. An asymptotic approach to the description of such electromagnetic radiation is proposed, in which the solutions of Maxwell's equations are expressed in terms of the solutions of a system of four ordinary differential equations and two algebraic equations for six components of the electromagnetic field in the zero approximation. The gradient of the phase front of the adiabatic guided mode satisfies the eikonal equation with respect to the effective refractive index of the waveguide for the given mode.

The multilayer structure of waveguides allows one more stage of reducing the model to a homogeneous system of linear algebraic equations, the nontrivial solvability condition of which specifies the relationship between the gradient of the radiation phase front and the gradients of interfaces between thin homogeneous layers.

In the final part of the work, eigenvalue and eigenvector problems (differential and algebraic), describing adiabatic guided modes are formulated. The formulation of the problem of describing the single-mode propagation of adiabatic guided modes is also given, emphasizing the adiabatic nature of the described approximate solution of Maxwell's equations.

Key words and phrases: smoothly irregular integrated optical multilayer waveguides, eigenvalue and eigenvector problem, single-mode propagation of adiabatic guided modes

\section{Introduction}

Fundamental results in the theory of regular waveguides were obtained for closed (metallic) waveguides by A. N. Tikhonov and A. A. Samarskii [1], and for open (dielectric) waveguides by A. G. Sveshnikov [2] and V. V. Shevchenko [3]. Among the irregular waveguides, one can distinguish transversely irregular and longitudinally irregular waveguides. For transversely irregular waveguides, the equations and the corresponding solutions allow the separation of variables [4].

(C) Sevastianov A. L., 2020 
Here the incomplete Galerkin method developed by A. G. Sveshnikov [2], [5], [6] received the greatest recognition.

For closed longitudinally irregular waveguides, B. Z. Katsenelenbaum developed the method of cross sections [7], which was generalized for open longitudinally irregular waveguides by V. V. Shevchenko [8]. These models do not describe depolarization and hybridization of guided modes in irregular sections of waveguides. A. A. Egorov, L. A. Sevastyanov and A. L. Sevastyanov developed the foundations of the theory of smoothly irregular 3D dielectric and, in particular, integrated optical waveguides [9], [10], which was successfully applied to a number of three-dimensional integrated optical waveguides and smoothly irregular 3D waveguide devices based on them [11]-[13]. The mathematical basis of the model of adiabatic guided modes (AGMs) is the asymptotic method and the method of coupled modes. The asymptotic method for solving a boundary value problem for a system of differential equations with respect to a small parameter $\delta$ allows it to be reduced to a system of ordinary differential equations with special boundary conditions, the method of solving which is known. The coupling of two second-order equations for modes of two different polarizations when solving the original system of equations by the asymptotic method, manifests itself in the first approximation as a weak (of the order of $\delta$ ) coupling of two linear oscillators. It reflects the violation of the structure regularity caused by a change in the phase constant of smoothly irregular dielectric waveguides.

In this paper, we consider an approach to the construction of a model of propagation of electromagnetic radiation in integrated optical smoothly irregular waveguide structures. Traditionally, such models are described using Maxwell's equations. The paper considers only monochromatic radiation, depending on time as $\exp (i \omega t)$. Such time dependence of the solution allows considering a model of steady-state guided propagation of electromagnetic radiation.

\section{Basic concepts and notations}

Guided propagation of monochromatic polarized electromagnetic radiation in integrated optical waveguides is described by Maxwell's equations. The electromagnetic field is described using complex amplitudes. A material medium is considered, consisting of dielectric subdomains that fill the entire three-dimensional space. The permittivities of the subdomains are different and real, and the permeability is everywhere equal to the permeability of vacuum. It follows that in the absence of foreign currents and charges, the induced currents and charges are equal to zero.

In the absence of foreign charges and currents, the scalar Maxwell's equations follow from the vector ones, and the boundary conditions for the normal components follow from the boundary conditions for the tangential components [14]. The constitutive equations are assumed to be linear. Thus, the electromagnetic field in a space filled with dielectrics in the Gaussian system of units is described by equations

$$
\operatorname{rot} \mathbf{E}=-\frac{1}{c} \frac{\partial \mathbf{B}}{\partial t}, \quad \operatorname{rot} \mathbf{H}=-\frac{1}{c} \frac{\partial \mathbf{D}}{\partial t}, \quad \mathbf{D}=\varepsilon \mathbf{E}, \quad \mathbf{B}=\mu \mathbf{H},
$$


where $\mathbf{E}, \mathbf{H}$ are the electric and magnetic field strength vectors, $\mathbf{D}$ is the electric displacement vector, $\mathbf{B}$ is the magnetic flux density vector, $c$ is the velocity of electromagnetic waves in vacuum.

At the interface between dielectric media 1 and 2, the tangential components of electric and magnetic field strengths satisfy the following boundary (matching) conditions:

$$
\left.\mathbf{H}_{\tau}\right|_{1}=\left.\mathbf{H}_{\tau}\right|_{2},\left.\quad \mathbf{E}_{\tau}\right|_{1}=\left.\mathbf{E}_{\tau}\right|_{2} .
$$

The asymptotic boundary conditions for guided modes at infinity

$$
\|\mathbf{E}\| \underset{|x| \rightarrow \infty}{\longrightarrow} 0, \quad\|\mathbf{H}\| \underset{|x| \rightarrow \infty}{\longrightarrow} 0,
$$

ensure uniqueness of the solution of the problem (1)-(3).

In equations (1), $\varepsilon$ is the medium permittivity, $\mu$ is the medium permeability. Let us denote by $n=\sqrt{\mu \varepsilon}$ the refractive index of the medium (hereinafter of a dielectric layer of the considered multilayer dielectric structure).

\section{The considered class of objects}

The object of our consideration is the guided propagation of monochromatic electromagnetic radiation of the optical range in thin-film integrated optical structures. Such structures are complex waveguide structures formed by the deposition of additional waveguide layers of various (smoothly irregular) geometric configurations on the base waveguide. As a base waveguide, we consider a regular planar three-layer waveguide filling the entire three-dimensional space (open waveguide): a substrate layer (substrate) is located in the lower half-space, then a guiding layer of constant thickness is located, and a cladding layer is located in the upper half-space. In this case, the guiding layer (core) is optically denser compared to the substrate and the cladding. By a thin-film waveguide, we mean a waveguide whose core thickness is comparable to the wavelength of propagating radiation.

Integrated optical waveguide structures are formed by introducing into the three-layer planar dielectric waveguide additional layers of variable thickness $h(y, z)$. The additional waveguide layers are specified by the interface between the additional and waveguide layers $x=h(y, z)$ and directly affect phase distribution $\varphi(y, z)$ and effective refractive index $n_{\text {eff }}(y, z)$ of the composite waveguide system. The phase velocity is smaller and the effective refractive index $n_{\text {eff }}(y, z)$ is greater in the locations where the total thickness of the waveguide layers is greater.

In particular, it can be a few-mode integrated optical waveguide implemented in the form of a three-layer dielectric "plate". On this structure, a local but smoothly irregular optical inhomogeneity is deposited, i.e., an additional dielectric layer of variable thickness. A thin-film waveguide generalized lens (TFWGL) (see Figure 1) is an example [11], [12]. 


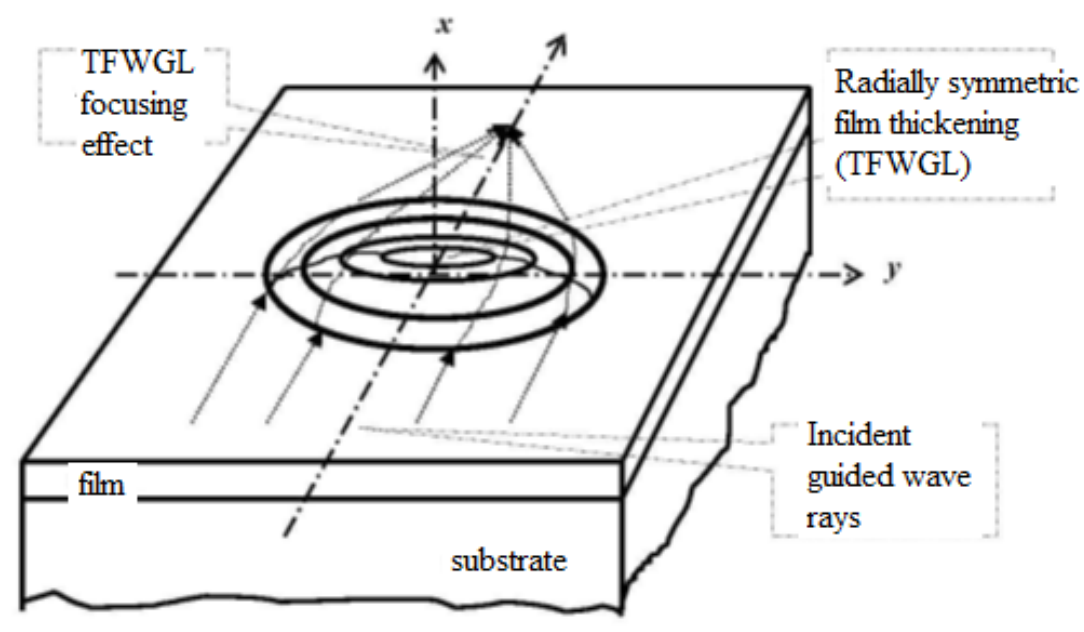

Figure 1. Schematic of Luneberg TFWGL with an additional waveguide layer having cylindrical symmetry

In such integrated optical waveguide, a normal mode of the planar waveguide (allowed by the structure ${ }^{1}$, see Appendix) travelling from infinity (or from a point source in the plane of the planar waveguide) is deformed when meeting the localized irregularity (optical inhomogeneity). However, it preserves the structure of a 'transverse resonance', i.e., the structure of a standing wave in the 'transverse' (vertical) direction. Gorelyshev, Neishtadt et al. [20], [21] formulate this conditions as conservation of an adiabatic invariant.

After passing the irregularity region, the deformed "adiabatically invariant" guided (quasi)mode restores the initial (vertical) parameters of a normal mode of a regular waveguide (or transforms into a superposition of normal modes).

For convenience, let us define the Cartesian coordinate system so that all spatial subdomains corresponding to infinite dielectric layers be bounded by planes parallel to the $y O z$-plane and surfaces asymptotically parallel to the $y O z$-plane, so that hereafter $\varepsilon=\varepsilon(x), \mu=1$.

We will call smoothly irregular the optical structures satisfying the inequalities specified by the geometry of the additional waveguide layer:

$$
\left|\frac{\partial h}{\partial y}\right|,\left|\frac{\partial h}{\partial z}\right| \ll 1
$$

In weakly inhomogeneous $3 \mathrm{D}$ media the electromagnetic radiation propagation is described by locally planar waves or adiabatic approximations to the solutions of the Maxwell's equations, obtained using the asymptotic method [22]. By analogy with locally plane and locally spherical 3D waves [22],

\footnotetext{
${ }^{1}$ From the theory of planar regular waveguides [15]-[19] it is known that an electromagnetic wave propagates through a regular waveguide in the form of a normal guided mode.
} 
[23], we seek the guided propagation of electromagnetic monochromatic polarized radiation in a smoothly irregular integrated optical waveguide in the form of modified locally normal guided modes of reference waveguides ${ }^{1}$.

The adiabatic approximation of the solution of Maxwell's equation obtained in this way will preserve the adiabatic invariants that reflect the guided character of light propagation (the so called transverse resonance condition [15], [26].

This work is devoted to the search for a model of adiabatic guided propagation of monochromatic electromagnetic radiation in smoothly irregular integrated optical structures. The technique for finding it is based on an asymptotic approach in the form of locally normal guided modes of a locally planar reference waveguide. The adiabatic approximation of the solution of Maxwell's equations obtained in this way will preserve adiabatic invariants reflecting the waveguide nature (the so-called transverse resonance condition) of light propagation.

\section{Basic equations of the adiabatic guided mode model}

Let us recall the earlier assumptions made for the considered integrated optical waveguides and the electromagnetic radiation propagating along them.

1. Electromagnetic radiation is optical and monochromatic with a fixed wavelength $\lambda \in[380 ; 780], \mathrm{nm}$.

2. The thickness of the guiding layer (core) of the base thin-film waveguide is comparable to the length of the propagating monochromatic electromagnetic radiation $d \sim \lambda$.

3 . The surface of additional waveguide layer $(x=h(y, z))$ satisfies the limiting conditions $\left|\frac{\partial h}{\partial y}\right|,\left|\frac{\partial h}{\partial z}\right| \ll 1$.

4. The integrated optical waveguide is a material medium consisting of dielectric subdomains that fill the entire space.

5. The permittivities of the subdomains are different and real, and the permeability is everywhere equal to the magnetic permeability of vacuum.

6. There are no external currents and charges. It follows from this that in the absence of external currents and charges, the induced currents and charges are equal to zero.

7. A Cartesian coordinate system is introduced as follows: the interfaces between the dielectric media of the basic three-layer waveguide are parallel to the $y O z$-plane. In this case, the subdomains of space corresponding to the cladding and substrate layers are semi-infinite, the additional waveguide layers are asymptotically parallel to the $y O z$-plane, so that $\varepsilon=\varepsilon(x)$.

In Cartesian coordinates associated with the geometry of the substrate (or a three-layer planar dielectric waveguide), Maxwell's equations are written in the form

\footnotetext{
${ }^{1}$ The notion of reference waveguides (dielectric planar) is presented in papers by Katsenelenbaum and Shevchenko [7], [8], [24], [25].
} 


$$
\begin{aligned}
\frac{\partial H_{z}}{\partial y}-\frac{\partial H_{y}}{\partial z}=\frac{\varepsilon}{c} \frac{\partial E_{x}}{\partial t}, & \frac{\partial E_{z}}{\partial y}-\frac{\partial E_{y}}{\partial z}=-\frac{\mu}{c} \frac{\partial H_{x}}{\partial t} \\
\frac{\partial H_{x}}{\partial z}-\frac{\partial H_{z}}{\partial x}=\frac{\varepsilon}{c} \frac{\partial E_{y}}{\partial t}, & \frac{\partial E_{x}}{\partial z}-\frac{\partial E_{z}}{\partial x}=-\frac{\mu}{c} \frac{\partial H_{y}}{\partial t} \\
\frac{\partial H_{y}}{\partial x}-\frac{\partial H_{x}}{\partial y}=\frac{\varepsilon}{c} \frac{\partial E_{z}}{\partial t}, & \frac{\partial E_{y}}{\partial x}-\frac{\partial E_{x}}{\partial y}=-\frac{\mu}{c} \frac{\partial H_{z}}{\partial t} .
\end{aligned}
$$

To construct the model of adiabatic guided modes (AGMs) we represent the solutions of (4) in the form of locally normal guided modes of a locally planar reference waveguide (see [7], [8], [27]), which in the method of asymptotic expansion take the form

$$
\begin{aligned}
& \vec{E}(x, y, z, t)=\sum_{s=0}^{\infty} \frac{\vec{E}_{s}(x, y, z)}{(-i \omega)^{\gamma+s}} \exp \left\{i \omega t-i k_{0} \varphi(y, z)\right\}, \\
& \vec{H}(x, y, z, t)=\sum_{s=0}^{\infty} \frac{\vec{H}_{s}(x, y, z)}{(-i \omega)^{\gamma+s}} \exp \left\{i \omega t-i k_{0} \varphi(y, z)\right\} .
\end{aligned}
$$

In the notation $\vec{E}_{s}(x, y, z, t), \vec{H}_{s}(x, y, z, t)$ the separation of $x$ with a semicolon means the following assumption

$$
\left\|\frac{\partial \vec{E}_{s}(x, y, z)}{\partial y}\right\|,\left\|\frac{\partial \vec{E}_{s}(x, y, z)}{\partial z}\right\| \sim \frac{1}{\omega}\left\|\frac{\partial \vec{E}_{s}(x, y, z)}{\partial x}\right\|, \quad j=x, y, z
$$

and

$$
\left\|\frac{\partial \vec{H}_{s}(x, y, z)}{\partial y}\right\|,\left\|\frac{\partial \vec{H}_{s}(x, y, z)}{\partial z}\right\| \sim \frac{1}{\omega}\left\|\frac{\partial \vec{H}_{s}(x, y, z)}{\partial x}\right\|, \quad j=x, y, z,
$$

where \|\| is the Euclidean norm and $\omega$ is the circular frequency of the propagating monochromatic electromagnetic radiation.

Using the approach of the asymptotic expansion method with respect to the dimensional small parameter $\omega^{-1}$ [23], [28], [29], we substitute expressions (5), (6) into the system of equations (4) and equate the coefficients at equal powers of the small parameter $\omega^{-1}$. As a result, with the relations (7) and (8) taken into account, in the zero approximation of the method of asymptotic expansion with respect to small parameter we arrive at a system of homogeneous equations:

$$
\begin{gathered}
-i k_{0} \frac{\partial \varphi}{\partial y} H_{0}^{z}+i k_{0} \frac{\partial \varphi}{\partial z} H_{0}^{y}=i k_{0} \varepsilon E_{0}^{x}, \\
-i k_{0} \frac{\partial \varphi}{\partial z} H_{0}^{x}-\frac{\partial H_{0}^{z}}{\partial x}=i k_{0} \varepsilon E_{0}^{y},
\end{gathered}
$$




$$
\begin{gathered}
\frac{\partial H_{0}^{y}}{\partial x}+i k_{0} \frac{\partial \varphi}{\partial y} H_{0}^{x}=i k_{0} \varepsilon E_{0}^{z} \\
-i k_{0} \frac{\partial \varphi}{\partial y} E_{0}^{z}+i k_{0} \frac{\partial \varphi}{\partial z} E_{0}^{y}=i k_{0} \mu H_{0}^{x} \\
-i k_{0} \frac{\partial \varphi}{\partial z} E_{0}^{x}-\frac{\partial E_{0}^{z}}{\partial x}=i k_{0} \mu H_{0}^{y} \\
\frac{\partial E_{0}^{y}}{\partial x}+i k_{0} \frac{\partial \varphi}{\partial y} E_{0}^{x}=i k_{0} \mu H_{0}^{z}
\end{gathered}
$$

By means of simple transformations, we reduce it to the form of interest to us. Namely, from the relation (12) we get the expression

$$
E_{0}^{x}=-\frac{\partial \varphi}{\partial y} \frac{1}{\varepsilon} H_{0}^{z}+\frac{\partial \varphi}{\partial z} \frac{1}{\varepsilon} H_{0}^{y}
$$

which we substitute into equations (13)-(14) that take the form

$$
\begin{aligned}
& -\frac{\partial E_{0}^{z}}{\partial x}=i k_{0} \frac{\partial \varphi}{\partial z}\left(-\frac{\partial \varphi}{\partial y} \frac{1}{\varepsilon} H_{0}^{z}+\frac{\partial \varphi}{\partial z} \frac{1}{\varepsilon} H_{0}^{y}\right)-i k_{0} \mu H_{0}^{y}, \\
& \frac{\partial E_{0}^{y}}{\partial x}=-i k_{0} \mu H_{0}^{z}-i k_{0} \frac{\partial \varphi}{\partial y}\left(-\frac{\partial \varphi}{\partial y} \frac{1}{\varepsilon} H_{0}^{z}+\frac{\partial \varphi}{\partial z} \frac{1}{\varepsilon} H_{0}^{y}\right) .
\end{aligned}
$$

From relation (9) we get the expression

$$
H_{0}^{x}=\frac{\partial \varphi}{\partial y} \frac{1}{\mu} E_{0}^{z}-\frac{\partial \varphi}{\partial z} \frac{1}{\mu} E_{0}^{y}
$$

and substitute it into equations (10)-(11), which take the form

$$
\begin{aligned}
-\frac{\partial H_{0}^{z}}{\partial x} & =-i k_{0} \varepsilon E_{0}^{y}+i k_{0} \frac{\partial \varphi}{\partial z}\left(\frac{\partial \varphi}{\partial y} \frac{1}{\mu} E_{0}^{z}-\frac{\partial \varphi}{\partial z} \frac{1}{\mu} E_{0}^{y}\right), \\
\frac{\partial H_{0}^{y}}{\partial x} & =i k_{0} \varepsilon E_{0}^{z}-i k_{0} \frac{\partial \varphi}{\partial y}\left(\frac{\partial \varphi}{\partial y} \frac{1}{\mu} E_{0}^{z}-\frac{\partial \varphi}{\partial z} \frac{1}{\mu} E_{0}^{y}\right) .
\end{aligned}
$$

Ultimately, Maxwell's equation reduced in the zero approximation to two algebraic equations and four differential equations of the first order.

At any fixed values of $(y, z)$, equations (15)-(16), (17)-(18) take the form of a system of ordinary differential equations of the first order. Hereinafter, we deal with the zero approximation of the asymptotic expansion with respect to the small parameter; therefore, the index of the order of smallness is omitted:

$$
\begin{aligned}
& \frac{d H^{z}}{d x}+\frac{i k_{0}}{\mu} \frac{\partial \varphi}{\partial z}\left(\frac{\partial \varphi}{\partial y} E^{z}-\frac{\partial \varphi}{\partial z} E^{y}\right)+i k_{0} \varepsilon E^{y}=0, \\
& \frac{d H^{y}}{d x}+\frac{i k_{0}}{\mu} \frac{\partial \varphi}{\partial y}\left(\frac{\partial \varphi}{\partial y} E^{z}-\frac{\partial \varphi}{\partial z} E^{y}\right)-i k_{0} \varepsilon E^{z}=0,
\end{aligned}
$$




$$
\begin{aligned}
& \frac{d E^{z}}{d x}+\frac{i k_{0}}{\varepsilon} \frac{\partial \varphi}{\partial z}\left(\frac{\partial \varphi}{\partial z} H^{y}-\frac{\partial \varphi}{\partial y} H^{z}\right)-i k_{0} \mu H^{y}=0, \\
& \frac{d E^{y}}{d x}+\frac{i k_{0}}{\varepsilon} \frac{\partial \varphi}{\partial y}\left(\frac{\partial \varphi}{\partial z} H^{y}-\frac{\partial \varphi}{\partial y} H^{z}\right)+i k_{0} \mu H^{z}=0 .
\end{aligned}
$$

In addition, for any fixed values of $(y, z)$ the algebraic equations are valid

$$
E_{0}^{x}=\frac{1}{\varepsilon}\left(\frac{\partial \varphi}{\partial z} H_{0}^{y}-\frac{\partial \varphi}{\partial y} H_{0}^{z}\right), \quad H_{0}^{x}=\frac{1}{\mu}\left(\frac{\partial \varphi}{\partial y} E_{0}^{z}-\frac{\partial \varphi}{\partial z} E_{0}^{y}\right) .
$$

Remark 1. In the proposed form (5)-(6) of the desired solutions of Maxwell's equations the quantities $\frac{\partial \varphi}{\partial y}$ and $\frac{\partial \varphi}{\partial z}$ have the meaning of phase constants of guided propagation of radiation in the directions $O y$ and $O z$ in all layers of the waveguide simultaneously, i.e., they determine the effective refractive index of the waveguide under study for the given adiabatic guided mode:

$$
\left(\frac{\partial \varphi}{\partial y}(y, z)\right)^{2}+\left(\frac{\partial \varphi}{\partial z}(y, z)\right)^{2}=n_{\mathrm{eff}}^{2}(y, z)
$$

From the analysis carried out, we conclude that with the accepted assumptions the zero approximation to the guided solution of Maxwell's equations is given by the following relations:

$$
\left\{\begin{array}{l}
\vec{E}(x, y, z, t) \\
\vec{H}(x, y, z, t)
\end{array}\right\}=\left\{\begin{array}{l}
\vec{E}_{0}(x ; y, z) \\
\vec{H}_{0}(x ; y, z)
\end{array}\right\} \exp \{i \omega t-i \varphi(y, z)\},
$$

with

$$
\begin{aligned}
& \varepsilon \frac{\partial E_{0}^{y}}{\partial x}=-i k_{0}\left(\frac{\partial \varphi}{\partial y}\right)\left(\frac{\partial \varphi}{\partial z}\right) H_{0}^{y}-i k_{0}\left(\varepsilon \mu-\left(\frac{\partial \varphi}{\partial y}\right)^{2}\right) H_{0}^{z} \\
& \varepsilon \frac{\partial E_{0}^{z}}{\partial x}=i k_{0}\left(\varepsilon \mu-\left(\frac{\partial \varphi}{\partial z}\right)^{2}\right) H_{0}^{y}+i k_{0}\left(\frac{\partial \varphi}{\partial z}\right)\left(\frac{\partial \varphi}{\partial y}\right) H_{0}^{z} \\
& \mu \frac{\partial H_{0}^{y}}{\partial x}=i k_{0}\left(\frac{\partial \varphi}{\partial y}\right)\left(\frac{\partial \varphi}{\partial z}\right) E_{0}^{y}+i k_{0}\left(\varepsilon \mu-\left(\frac{\partial \varphi}{\partial y}\right)^{2}\right) E_{0}^{z} \\
& \mu \frac{\partial H_{0}^{z}}{\partial x}=-i k_{0}\left(\varepsilon \mu-\left(\frac{\partial \varphi}{\partial z}\right)^{2}\right) E_{0}^{y}-i k_{0}\left(\frac{\partial \varphi}{\partial z}\right)\left(\frac{\partial \varphi}{\partial y}\right) E_{0}^{z}
\end{aligned}
$$

and

$$
E_{0}^{x}=-\frac{\partial \varphi}{\partial y} \frac{1}{\varepsilon} H_{0}^{z}+\frac{\partial \varphi}{\partial z} \frac{1}{\varepsilon} H_{0}^{y}, \quad H_{0}^{x}=\frac{\partial \varphi}{\partial y} \frac{1}{\mu} E_{0}^{z}-\frac{\partial \varphi}{\partial z} \frac{1}{\mu} E_{0}^{y}
$$

as well as

$$
\left(\frac{\partial \varphi}{\partial y}(y, z)\right)^{2}+\left(\frac{\partial \varphi}{\partial z}(y, z)\right)^{2}=n_{\mathrm{eff}}^{2}(y, z)
$$


For thin-film multilayer waveguide, consisting of optically homogeneous layers, from (2) the conditions of the electromagnetic field matching at the interfaces between the media follow:

$$
\begin{aligned}
& \vec{n} \times \vec{E}^{-}+\vec{n} \times \vec{E}^{+}=0, \\
& \vec{n} \times \vec{H}^{-}+\vec{n} \times \vec{H}^{+}=0 .
\end{aligned}
$$

From (3) the asymptotic conditions follow

$$
E_{y}^{0}, E_{z}^{0}, H_{y}^{0}, H_{z}^{0} \underset{x \rightarrow \pm \infty}{\longrightarrow} 0 .
$$

The system of equations (20), (24) for any fixed $(y, z)$ defines a problem of finding eigenvalues $(\vec{\nabla} \varphi)_{j}^{2}(y, z)$ and eigenfunctions $\left(E_{y}^{j}, E_{z}^{j}, H_{y}^{j}, H_{z}^{j}\right)^{T}(y, z)$, normalized to unity:

$$
\int_{-\infty}^{\infty}\left|E_{y}^{j}\right|^{2} d x=1, \quad \int_{-\infty}^{\infty}\left|H_{y}^{j}\right|^{2} d x=1 .
$$

\section{Algebraic model of adiabatic guided modes}

In the case of a multilayer integrated optical waveguide consisting of homogeneous dielectric layers (possible, with complex permittivities) the relations (20), (22)-(24) are valid with generally non-horizontal interfaces between the layers (see Figure 2).

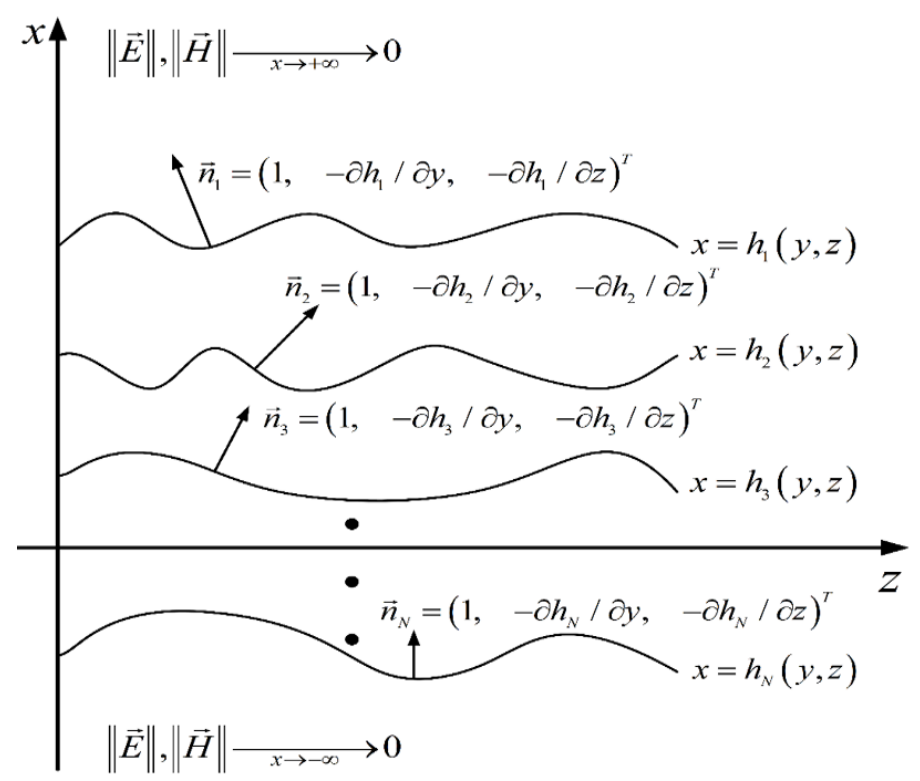

Figure 2. Structure of a multilayer thin-film waveguide 
In this case, in each inner layer the four-dimensional system of ordinary differential equations with constant coefficients has a four-parameter system of fundamental solutions. Then the general solutions of equations (20) in the layers take the form

$$
\begin{gathered}
\vec{U}(x ; y, z)=C_{1} \vec{\xi}_{1} e^{\gamma_{+} x}+C_{2} \vec{\xi}_{2} e^{\gamma_{-} x}+C_{3} \vec{\xi}_{3} e^{\gamma_{+} x}+C_{4} \vec{\xi}_{4} e^{\gamma_{-} x}, \\
E_{y}(x)=\frac{-I\left(A_{2}-A_{4}\right) \sqrt{-\mu \varepsilon+\frac{\partial \varphi^{2}}{\partial y}+\frac{\partial \varphi^{2}}{\partial z}} \mu \exp \left\{-k_{0} \sqrt{\frac{\partial \varphi^{2}}{\partial y}+\frac{\partial \varphi^{2}}{\partial z}-\mu \varepsilon x}\right\}}{\frac{\partial \varphi}{\partial y} \frac{\partial \varphi}{\partial z}}- \\
-\frac{\exp \left\{k_{0} \sqrt{\frac{\partial \varphi^{2}}{\partial y}+\frac{\partial \varphi^{2}}{\partial z}-\mu \varepsilon x}\right\}\left(A_{1}+A_{3}\right)\left(\mu \varepsilon-\frac{\partial \varphi^{2}}{\partial y}\right)}{\frac{\partial \varphi}{\partial y} \frac{\partial \varphi}{\partial z}}, \\
H_{z}(x)=\frac{\frac{\partial \varphi}{\partial y} \frac{\partial \varphi}{\partial z}}{-\left(A_{2}+A_{4}\right)\left(\mu \varepsilon-\frac{\partial \varphi^{2}}{\partial z}\right) \exp \left\{-k_{0} \sqrt{\frac{\partial \varphi^{2}}{\partial y}+\frac{\partial \varphi^{2}}{\partial z}-\mu \varepsilon x}\right\}}- \\
-\frac{\left.I_{1}-A_{4}\right) \varepsilon \exp \left\{k_{0} \sqrt{\frac{\partial \varphi^{2}}{\partial y}+\frac{\partial \varphi^{2}}{\partial z}-\mu \varepsilon x}\right\} \sqrt{\frac{\partial \varphi^{2}}{\partial y} \frac{\partial \varphi^{2}}{\partial z}-\mu \varepsilon}}{\frac{\partial \varphi}{\partial y} \frac{\partial \varphi}{\partial z}} \\
H_{y}(x)=\left(A_{2}+A_{4}\right) \exp \left\{-k_{0} \sqrt{\frac{\partial \varphi^{2}}{\partial y}+\frac{\partial \varphi^{2}}{\partial z}-\mu \varepsilon x}\right\} \\
E_{z}(x)=\left(A_{1}+A_{3}\right) \exp \left\{k_{0} \sqrt{\frac{\partial \varphi^{2}}{\partial y}+\frac{\partial \varphi^{2}}{\partial z}-\mu \varepsilon x}\right\}
\end{gathered}
$$

where

$$
\vec{u}=\left(H^{z}(x ; y, z) ; E^{z}(x ; y, z) ; H^{y}(x ; y, z) ; E^{y}(x ; y, z)\right)^{T}
$$

and $\gamma_{ \pm}= \pm k_{0} \sqrt{-\varepsilon \mu+\varphi_{y}^{2}+\varphi_{z}^{2}}$.

The rest components of the electromagnetic field are calculated using formulas (21), and all the six components enter the explicit form of relations (22), (23).

In the substrate and cladding layers due to asymptotic conditions the two-dimensional systems of ordinary differential equations with constant coefficients have two-parametric systems of fundamental solutions.

The solution unique for all layers satisfies the matching conditions at the interfaces between the layers, i.e., determines a system of linear algebraic equations for indefinite coefficients, which specify the expression of particular solutions in the layers in terms of the systems of fundamental solutions. 
At an arbitrary smooth interface between two dielectric media, described by the equation $F(x, y, z)=x-h(y, z)=0$ (see Figure 3), conditions (22) and (23) take the form

$$
\begin{aligned}
& {[\vec{n} \times \vec{E}]=\left(E_{y} \frac{\partial h}{\partial z}-E_{z} \frac{\partial h}{\partial y} ;-E_{z}-E_{x} \frac{\partial h}{\partial z} ; E_{y}-E_{x} \frac{\partial h}{\partial y}\right)^{T},} \\
& {[\vec{n} \times \vec{H}]=\left(H_{y} \frac{\partial h}{\partial z}-H_{z} \frac{\partial h}{\partial y} ;-H_{z}-H_{x} \frac{\partial h}{\partial z} ; H_{y}-H_{x} \frac{\partial h}{\partial y}\right)^{T} .}
\end{aligned}
$$

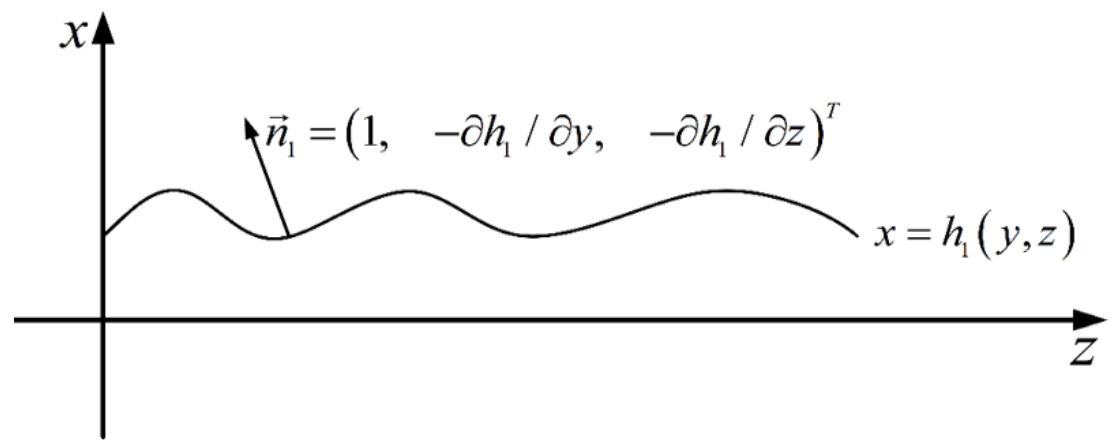

Figure 3. Equation of a normal to the interface between the layers

In the expressions $(25)$ and $(26)$ the denominator $\sqrt{1+\left(\frac{\partial h}{\partial y}\right)^{2}+\left(\frac{\partial h}{\partial z}\right)^{2}}$ in the expressions for the normal was omitted, since it is nonzero and coincides in both sides of equations (25), (26). It is worth noting that only two of three components of the obtained vectors (25) and (26) are linearly independent. Therefore, for writing the boundary conditions we will use the following expressions:

$$
\begin{aligned}
& \left.\left(A_{z}^{1}+A_{x}^{1} \frac{\partial h}{\partial z}\right)\right|_{x=h(y, z)}=\left.\left(A_{z}^{2}+A_{x}^{2} \frac{\partial h}{\partial z}\right)\right|_{x=h(y, z)}, \\
& \left.\left(A_{y}^{1}-A_{x}^{1} \frac{\partial h}{\partial y}\right)\right|_{x=h(y, z)}=\left.\left(A_{y}^{2}-A_{x}^{2} \frac{\partial h}{\partial y}\right)\right|_{x=h(y, z)},
\end{aligned}
$$

where $\vec{A}=\{\vec{E}, \vec{H}\}$. Moreover, for a planar boundary $F(x, y, z)=x-$ const the above expressions simplify to the following form:

$$
\left.A_{y}^{1}\right|_{x=\text { const }}=\left.A_{y}^{2}\right|_{x=\text { const }},\left.\quad A_{z}^{1}\right|_{x=\text { const }}=\left.A_{z}^{2}\right|_{x=\text { const }} .
$$

The above relations should be completed with the asymptotic condition (24).

Thus, in each inner layer of a $k$-layer waveguide the four-dimensional general solution is parametrized by four indefinite coefficients, in the substrate and cladding layers at the expense of asymptotic conditions the number of coefficients is reduced by two in each layer. Therefore, there is a total of $4(k-2)+2+2=4(k-1)$ coefficients. To each of $(k-1)$ interfaces four 
equations correspond, making the total of $4(k-1)$. Thus, we have $4(k-1)$ linear homogeneous algebraic equations for $4(k-1)$ unknown coefficients.

Returning to the calculation of the electromagnetic field in the multilayer waveguide, we seek a nontrivial solution to the homogeneous system of linear algebraic equations with respect to the coefficient of expansion over the fundamental system of solutions in each dielectric layer:

$$
M(h(y, z), \nabla h(y, z), \varphi(y, z), \nabla \varphi(y, z)) \vec{A}=\overrightarrow{0} .
$$

To find nontrivial fields $\vec{E}, \vec{H}$ at an arbitrary point $(y, z)$ the condition of solvability should be satisfied for the system of homogeneous linear algebraic equations

$$
\operatorname{det}(M(h(y, z), \nabla h(y, z), \varphi(y, z), \nabla \varphi(y, z)))=0 .
$$

Thus the system of homogeneous linear algebraic equations (27), nontrivially solvable under the condition (28), is an algebraic model of adiabatic guided modes in a smoothly irregular multilayer integrated optical waveguide. The roots of equation (28) are a set of eigenvalues and the solution of the system of equations (27) after substitution of each particular root is the corresponding eigenvector normalized by the condition $\|\vec{A}\|=1$.

\section{Results}

Thus, in the course of several stages of sequential reduction, we have formulated a number of problems of modeling the adiabatic guided modes in a smoothly irregular integrated optical multilayer waveguide.

Problem 1. The problem of finding eigenvalues and eigenfunctions within the AGM model

$$
\left\{\begin{array}{l}
\vec{E}(x, y, z, t) \\
\vec{H}(x, y, z, t)
\end{array}\right\}=\left\{\begin{array}{l}
\vec{E}_{0}(x ; y, z) \\
\vec{H}_{0}(x ; y, z)
\end{array}\right\} \exp \{i \omega t-i \varphi(y, z)\},
$$

is formulated as follows:

$$
\begin{gathered}
\varepsilon \frac{\partial E_{0}^{y}}{\partial x}=-i k_{0}\left(\frac{\partial \varphi}{\partial y}\right)\left(\frac{\partial \varphi}{\partial z}\right) H_{0}^{y}-i k_{0}\left(\varepsilon \mu-\left(\frac{\partial \varphi}{\partial y}\right)^{2}\right) H_{0}^{z} \\
\varepsilon \frac{\partial E_{0}^{z}}{\partial x}=i k_{0}\left(\varepsilon \mu-\left(\frac{\partial \varphi}{\partial z}\right)^{2}\right) H_{0}^{y}+i k_{0}\left(\frac{\partial \varphi}{\partial z}\right)\left(\frac{\partial \varphi}{\partial y}\right) H_{0}^{z} \\
\mu \frac{\partial H_{0}^{y}}{\partial x}=i k_{0}\left(\frac{\partial \varphi}{\partial y}\right)\left(\frac{\partial \varphi}{\partial z}\right) E_{0}^{y}+i k_{0}\left(\varepsilon \mu-\left(\frac{\partial \varphi}{\partial y}\right)^{2}\right) E_{0}^{z} \\
\mu \frac{\partial H_{0}^{z}}{\partial x}=-i k_{0}\left(\varepsilon \mu-\left(\frac{\partial \varphi}{\partial z}\right)^{2}\right) E_{0}^{y}-i k_{0}\left(\frac{\partial \varphi}{\partial z}\right)\left(\frac{\partial \varphi}{\partial y}\right) E_{0}^{z}
\end{gathered}
$$


Eigenfunctions $\left(E_{y}^{0}, E_{z}^{0}, H_{y}^{0}, H_{z}^{0}\right)_{j}^{T}\left(x ;(\nabla \varphi)_{j}^{2}\right)$ correspond to eigenvalues $(\nabla \varphi)_{j}^{2}$

The accompanying components of the electromagnetic field are calculated by the formulas:

$$
E_{0}^{x}=-\frac{\partial \varphi}{\partial y} \frac{1}{\varepsilon} H_{0}^{z}+\frac{\partial \varphi}{\partial z} \frac{1}{\varepsilon} H_{0}^{y}, \quad H_{0}^{x}=\frac{\partial \varphi}{\partial y} \frac{1}{\mu} E_{0}^{z}-\frac{\partial \varphi}{\partial z} \frac{1}{\mu} E_{0}^{y} .
$$

Problem 2. The problem of finding eigenvalues of eigenfunctions within the AGM model

$$
\left\{\begin{array}{l}
\vec{E}(x, y, z, t) \\
\vec{H}(x, y, z, t)
\end{array}\right\}=\left\{\begin{array}{l}
\vec{E}_{0}(x ; y, z) \\
\vec{H}_{0}(x ; y, z)
\end{array}\right\} \exp \{i \omega t-i \varphi(y, z)\},
$$

is formulated as follows:

$$
\frac{\partial^{2} E_{0}^{y}}{\partial x^{2}}+k_{0}^{2}\left(\varepsilon \mu-(\nabla \varphi)^{2}\right) E_{0}^{y}=0, \quad \frac{\partial^{2} H_{0}^{y}}{\partial x^{2}}+k_{0}^{2}\left(\varepsilon \mu-(\nabla \varphi)^{2}\right) H_{0}^{y}=0 .
$$

Eigenfunctions $\left(E_{y}^{0}, H_{y}^{0},\right)_{j}^{T}\left(x ;(\nabla \varphi)_{j}^{2}\right)$ correspond to eigenvalues $(\nabla \varphi)_{j}^{2}$.

The accompanying components of the electromagnetic field are calculated by the formulas

$$
\begin{gathered}
\varepsilon \frac{\partial E_{0}^{y}}{\partial x}=-i k_{0}\left(\frac{\partial \varphi}{\partial y}\right)\left(\frac{\partial \varphi}{\partial z}\right) H_{0}^{y}-i k_{0}\left(\varepsilon \mu-\left(\frac{\partial \varphi}{\partial y}\right)^{2}\right) H_{0}^{z} \\
\varepsilon \frac{\partial E_{0}^{z}}{\partial x}=i k_{0}\left(\varepsilon \mu-\left(\frac{\partial \varphi}{\partial z}\right)^{2}\right) H_{0}^{y}+i k_{0}\left(\frac{\partial \varphi}{\partial z}\right)\left(\frac{\partial \varphi}{\partial y}\right) H_{0}^{z} \\
\mu \frac{\partial H_{0}^{y}}{\partial x}=i k_{0}\left(\frac{\partial \varphi}{\partial y}\right)\left(\frac{\partial \varphi}{\partial z}\right) E_{0}^{y}+i k_{0}\left(\varepsilon \mu-\left(\frac{\partial \varphi}{\partial y}\right)^{2}\right) E_{0}^{z} \\
\mu \frac{\partial H_{0}^{z}}{\partial x}=-i k_{0}\left(\varepsilon \mu-\left(\frac{\partial \varphi}{\partial z}\right)^{2}\right) E_{0}^{y}-i k_{0}\left(\frac{\partial \varphi}{\partial z}\right)\left(\frac{\partial \varphi}{\partial y}\right) E_{0}^{z}
\end{gathered}
$$

Problem 3. Following the ideology of the cross section method, the steadystate regime of guided propagation of electromagnetic radiation within the frameworks of the AGM model, i.e., the solution of Maxwell's equations rot $\vec{H}=$ $i k \varepsilon \vec{E}, \operatorname{rot} \vec{E}=-i k \mu \vec{H}$ with asymptotic conditions $(\vec{E}, \vec{H})^{T}(x) \underset{x \rightarrow \pm \infty}{\longrightarrow} \overrightarrow{0}$ is sought in the form of a sum

$$
(\vec{E}, \vec{H})^{T}(x, y, z)=\sum_{j} C_{\vec{\beta}_{j}}(y, z)(\vec{E}, \vec{H})^{T}\left(x, \vec{\beta}_{j}\right),
$$


where $(\vec{E}, \vec{H})^{T}\left(x, \vec{\beta}_{j}\right)$ are solutions to the equations (30)-(31), (32) with additional condition:

$$
\vec{\nabla}(\vec{E}, \vec{H})^{T}(x, y, z)=-\sum_{j} i \beta_{j} C_{\vec{\beta}_{j}}(y, z)(\vec{E}, \vec{H})^{T}\left(x, \vec{\beta}_{j}\right),
$$

where $\vec{\beta}(y, z)=\vec{\nabla} \varphi(y, z)$.

Problem 4. Following the ideology of the method of cross sections, the steady-state regime of guided propagation of electromagnetic radiation within the frameworks of the AGM model, i.e., the solution of Maxwell's equations $\operatorname{rot}(\operatorname{rot} \vec{H})=k_{0}^{2} \varepsilon \mu \vec{E}, \operatorname{rot}(\operatorname{rot} \vec{E})=k_{0}^{2} \varepsilon \mu \vec{H}$ with asymptotic conditions $(\vec{E}, \vec{H})^{T}(x) \underset{x \rightarrow \pm \infty}{\longrightarrow} \overrightarrow{0}$ is sought in the form of a sum

$$
(\vec{E}, \vec{H})^{T}(x, y, z)=\sum_{j} C_{\vec{\beta}_{j}}(y, z)(\vec{E}, \vec{H})^{T}\left(x, \vec{\beta}_{j}\right),
$$

where $(\vec{E}, \vec{H})^{T}\left(x, \vec{\beta}_{j}\right)$ are solutions to the equations (33), (34) with additional condition:

$$
\vec{\nabla}(\vec{E}, \vec{H})^{T}(x, y, z)=-\sum_{j} i \beta_{j} C_{\vec{\beta}_{j}}(y, z)(\vec{E}, \vec{H})^{T}\left(x, \vec{\beta}_{j}\right),
$$

where $\vec{\beta}(y, z)=\vec{\nabla} \varphi(y, z)$.

The single-mode regime is specified by ansatz

$$
(\vec{E}, \vec{H})_{j}^{T}(x, y, z)=C_{\vec{\beta}_{j}}(y, z)(\vec{E}, \vec{H})^{T}\left(x, \vec{\beta}_{j}\right)
$$

with the additional condition

$$
\vec{\nabla}(\vec{E}, \vec{H})_{j}^{T}(x, y, z)=-i \vec{\beta}_{j} C_{\vec{\beta}_{j}}(y, z)(\vec{E}, \vec{H})^{T}\left(x, \vec{\beta}_{j}\right),
$$

where $\vec{\beta}(y, z)=\vec{\nabla} \varphi(y, z)$.

Remark 2. Similar to the method of cross sections [24], [25], [30], the solution of the last equation has the form

$$
C_{\vec{\beta}_{j}^{2}}(y, z)=\frac{1}{\beta(y, z)} \exp \left\{i k_{0} \int_{y_{0}, z_{0}}^{y, z}\left(\beta_{y}(y, z) d y+\beta_{z}(y, z) d z\right)\right\},
$$

which together with relations $(29),(30)-(31),(32)$ concludes the description of the adiabatic character of the model under consideration. 


\section{Conclusion}

The development of methods for the rigorous and approximate analysis of smoothly irregular integrated optical waveguides requires the development of new mathematical models of the corresponding objects, as well as the use of new methods for studying the problems arising in this case. The fundamental problem of electrodynamics of smoothly irregular waveguide three-dimensional (3D) structures is the development of stable methods and algorithms for solving the corresponding Maxwell's equations.

The paper considers an approach to the formulation of the problem of propagation of electromagnetic radiation in integrated optical smoothly irregular waveguide structures. Traditionally, similar problems are formulated based on Maxwell's equations. In this paper, only monochromatic radiation is considered, which is reflected in the dependence of solution on the frequency of propagating radiation. This type of time dependence of the solution allows considering a steady-state electrodynamic problem for electromagnetic radiation.

The problem of finding the eigenvectors (guided modes) and eigenvalues is considered using the model of adiabatic guided modes in the framework of the zero approximation of the asymptotic expansion for a planar regular threelayer optical waveguide. Considering that the permittivity and permeability are piecewise constant functions, the problem is solved in each subdomain with constant values of $\varepsilon, \mu$ with subsequent matching of solutions at the interfaces between the dielectric media. In each layer $\varepsilon, \mu$ have constant values, and the construction of the entire fields $\vec{E}, \vec{H}$ requires setting and solving the problem of finding $\varphi(y, z)$.

The paper considers a class of smoothly irregular integrated-optical multilayer waveguides, the properties of which determine the characteristic features of waveguide propagation of monochromatic polarized light in them. An asymptotic approach to the description of this type of electromagnetic radiation is proposed, reducing the solutions of the system of Maxwell's equations to a form, which is expressed in terms of the solutions of a system of four ordinary differential equations and two algebraic equations for six components of the electromagnetic field in the zero approximation.

The multilayer structure of waveguides allows one more stage of reducing the model to a homogeneous system of linear algebraic equations, the nontrivial solvability condition of which specifies the relationship between the gradient of the phase front of radiation and the gradients of interfaces between thin homogeneous layers.

In the final part of the work, eigenvalues and eigenvector problems (differential and algebraic) describing adiabatic guided modes are formulated. The problem of describing the single-mode propagation of adiabatic guided modes is also formulated with emphasis on the adiabatic nature of the described approximate solution of Maxwell's equations.

\section{Acknowledgments}

The publication has been prepared with the support of the Russian Foundation for Basic Research (RFBR) according to the research project No 19-01-00645. 


\section{Appendix. Normal modes of a regular planar optical waveguide}

\section{"Plane" guided modes}

The monographic literature [15]-[19] widely describes normal modes of a regular planar dielectric waveguide propagating along the axis $O z$ from $-\infty$ to $\infty$ (and/or back). In the vertical direction (along the $O x$ axis) they have the structure of standing waves, while in the horizontal direction along the waveguide they are travelling waves and are considered (not quite correctly) steady-state ('invariant') in the direction transverse with respect to the propagation direction (along the axis $O y$ ).

The vertical distribution of the electromagnetic field of a TE mode expressed in terms of the 'leading' transverse component of the electric field $E_{y}$ is given by the equation

$$
\frac{d^{2} E_{y}}{d x^{2}}(x)+\left(\varepsilon \mu-\beta^{2}\right) E_{y}(x)=0 .
$$

Two other components of the electromagnetic field of the $T E$ mode are expressed in terms of the leading one by the formulas

$$
H_{x}=-\frac{\beta}{\mu} E_{y}, \quad H_{z}=\frac{1}{i k_{0} \mu} \frac{d E_{y}}{d x} .
$$

These three relations can be derived from Maxwell's equations in the form (4).

For $T M$ modes analogous relations that follow from Maxwell's equations have the form

$$
\begin{gathered}
\varepsilon \frac{d}{d x}\left(\frac{1}{\varepsilon} \frac{d H_{y}}{d x}\right)(x)+\left(\varepsilon \mu-\beta^{2}\right) H_{y}(x)=0 \\
E_{x}=\frac{\beta}{\varepsilon} H_{y}, \quad E_{z}=-\frac{1}{i k_{0} \varepsilon} \frac{d H_{y}}{d x} .
\end{gathered}
$$

Equations (35) and (37) in multilayer waveguides composed of uniform regular dielectric layers, in each subdomain of the real axis (at the intersection of each layer with the vertical axis) take the form of second-order linear ordinary differential equations with constant coefficients. Therefore, the most common form of solutions to these equations is obtained using a fundamental system of solutions. Consequently, the distributions of the corresponding leading components of the electromagnetic field are written in the form of expansions of general solutions in terms of $(\sin (\chi x), \cos (\chi x))$ or $(\exp \{ \pm i(\chi x)\})$ and the expansion coefficients of the particular solution are determined from the boundary conditions (22)-(23).

These solutions, in particular, can be obtained according to the following algorithm. 
For guided $T E$ modes we get a homogeneous system of linear algebraic equations (SLAE) with matrix $\mathbf{M}_{T E}^{\perp 4}(\beta)$ with respect to variables $A_{s}^{+}, A_{1}^{+}, A_{1}^{+}, A_{c}^{-}$

$$
\begin{gathered}
A_{s}^{+} \exp \left\{\gamma_{s}^{j} a_{1}\right\}=A_{1}^{+} \exp \left\{i \chi_{1}^{j} a_{1}\right\}+A_{1}^{-} \exp \left\{-i \chi_{1}^{j} a_{1}\right\}, \\
\frac{\gamma_{s}^{j}}{i k_{0}} A_{s}^{+} \exp \left\{\gamma_{s}^{j} a_{1}\right\}=\frac{\chi_{1}^{j}}{k_{0}}\left(A_{1}^{+} \exp \left\{i \chi_{1}^{j} a_{1}\right\}-A_{1}^{-} \exp \left\{-i \chi_{1}^{j} a_{1}\right\}\right), \\
A_{1}^{+} \exp \left\{i \chi_{1}^{j} a_{2}\right\}+A_{1}^{-} \exp \left\{-i \chi_{1}^{j} a_{2}\right\}=A_{c}^{-} \exp \left\{-\gamma_{c}^{j} a_{2}\right\}, \\
\frac{\chi_{1}^{j}}{k_{0}}\left(A_{1}^{+} \exp \left\{i \chi_{1}^{j} a_{2}\right\}-A_{1}^{-} \exp \left\{-i \chi_{1}^{j} a_{2}\right\}\right)=-\frac{\gamma_{c}^{j}}{i k_{0}} A_{c}^{-} \exp \left\{-\gamma_{c}^{j} a_{2}\right\} .
\end{gathered}
$$

The homogeneous SLAE $\left(\hat{M}_{E}\right) \vec{A}=\overrightarrow{0}$ is nontrivially solvable if and only if its determinant is zero,

$$
\operatorname{det}\left(\hat{M}_{E}\right)=0
$$

For guided $T M$ modes a system of homogeneous linear algebraic equations is obtained with the matrix $\mathbf{M}_{T M}^{\perp 4}(\beta)$ for unknowns $B_{s}^{+}, B_{1}^{+}, B_{1}^{+}, B_{c}^{-}$, the solutions of which yield the values of the unknown amplitude coefficients

$$
\begin{gathered}
B_{s}^{+} \exp \left\{\gamma_{s}^{j} a_{1}\right\}=B_{1}^{+} \exp \left\{i \chi_{1}^{j} a_{1}\right\}+B_{1}^{-} \exp \left\{-i \chi_{1}^{j} a_{1}\right\}, \\
\frac{\gamma_{s}^{j}}{i k_{0} \varepsilon_{s}} B_{s}^{+} \exp \left\{\gamma_{s}^{j} a_{1}\right\}=\frac{\chi_{1}^{j}}{k_{0} \varepsilon_{1}}\left(B_{1}^{+} \exp \left\{i \chi_{1}^{j} a_{1}\right\}-B_{1}^{-} \exp \left\{-i \chi_{1}^{j} a_{1}\right\}\right), \\
B_{1}^{+} \exp \left\{i \chi_{1}^{j} a_{2}\right\}+B_{1}^{-} \exp \left\{-i \chi_{1}^{j} a_{2}\right\}=B_{c}^{-} \exp \left\{-\gamma_{c}^{j} a_{2}\right\}, \\
\frac{\chi_{1}^{j}}{k_{0} \varepsilon_{1}}\left(B_{1}^{+} \exp \left\{i \chi_{1}^{j} a_{2}\right\}-B_{1}^{-} \exp \left\{-i \chi_{1}^{j} a_{2}\right\}\right)=-\frac{\gamma_{c}^{j}}{i k_{0} \varepsilon_{c}} B_{c}^{-} \exp \left\{-\gamma_{c}^{j} a_{2}\right\} .
\end{gathered}
$$

The homogeneous SLAE $\left(\hat{M}_{H}\right) \vec{B}=\overrightarrow{0}$ is nontrivially solvable if its determinant equals zero

$$
\operatorname{det}\left(\hat{M}_{H}\right)=0 \text {. }
$$

Here $A$ and $B$ are the coefficients of expansion of $T E$ and $T M$ modes in terms of the fundamental system of solutions, respectively.

Equations (39) and (40) are equivalent to the dispersion relations in the trigonometric form

$$
\chi_{f} d=\operatorname{arctg}\left(\frac{\gamma_{c}^{m}}{\chi_{1}^{m}}\right)+\operatorname{arctg}\left(\frac{\gamma_{s}^{m}}{\chi_{1}^{m}}\right)+m \pi
$$

for guided $T E$ modes and

$$
\chi_{f} d=\operatorname{arctg}\left(\frac{\varepsilon_{1} \gamma_{c}^{m}}{\varepsilon_{c} \chi_{1}^{m}}\right)+\operatorname{arctg}\left(\frac{\varepsilon_{1} \gamma_{s}^{m}}{\varepsilon_{s} \chi_{1}^{m}}\right)+m \pi
$$

for guided $T M$ modes. 
If the expressions for the electromagnetic field strength are presented in complex form with the described dependence on the rest coordinates and time taken into account, i.e., in the form

$$
\begin{gathered}
\left(E_{y}, H_{x}, H_{z}\right)^{T}(x, y, z, t)=\left(A_{1}, A_{2}, A_{3}\right)^{T}(x, y, z) \exp \left\{i \omega t-i \varepsilon_{E}(x, y, z)\right\}, \\
\left(H_{y}, E_{x}, E_{z}\right)^{T}(x, y, z, t)=\left(B_{1}, B_{2}, B_{3}\right)^{T}(x, y, z) \exp \left\{i \omega t-i \varepsilon_{H}(x, y, z)\right\}
\end{gathered}
$$

with real-valued amplitude $A(B)$ and phase $\varphi_{E}\left(\varphi_{H}\right)$, then the phase remains constant in time along $x$, defines a travelling wave along $z$, and is constant along $y$. In other words, the phase front of the described solution to Maxwell's equations is "planar" (i.e., linear in the $y O z$-plane) and defines a "plane" ( i.e., linear in the $y O z$-plane) wave. There exist "forward" and "backward" waves travelling in opposite directions along the $z$-axis. The can be identified with the "plane" (in the $y O z$-plane) waves emitted by infinitely remote sources.

\section{"Cylindrical" guided modes}

Now let us proceed to the guided modes of a regular planar dielectric waveguide, excited by a source linear along the $O x$-axis and point-like in the $y O z$-plane, localized at the point $\left(0, z_{0}\right)$. The structure of the modes along the $O x$-axis completely coincides with that of "plane" guided and leaky $T E$ and $T M$ modes. Let us analyze the structure of cylindrical guided modes propagating in the $y O z$-plane

$$
\left(\begin{array}{l}
\vec{E} \\
\vec{H}
\end{array}\right)(x, y, z, t)=\left(\begin{array}{l}
\vec{E} \\
\vec{H}
\end{array}\right)_{\beta}^{E, H}(x) \exp \left\{i \omega t-i k_{0} \beta r\right\},
$$

where $r^{2}=y^{2}+\left(z-z_{0}\right)^{2} ; y=r \sin \theta, z=z_{0}+r \cos \theta$. In the $y O z$-plane circular fronts propagate from the origin of polar coordinates $y=r \sin \theta$, $z=z_{0}+r \cos \theta$.

Thus, in both cases the solutions for the normal modes are written as

$$
\left(\begin{array}{c}
\vec{E} \\
\vec{H}
\end{array}\right)(x, y, z, t)=\left(\begin{array}{l}
\vec{E} \\
\vec{H}
\end{array}\right)_{\beta}^{E, H}(x) \frac{\exp \left\{i \omega t-i k_{0} \varphi(y, z)\right\}}{\sqrt{\beta^{E, H}}},
$$

where:

$-\varphi(y, z)=\beta z$ for the modes from an infinitely remote source and

$-\varphi(y, z)=\beta \sqrt{y^{2}+\left(z-z_{0}\right)^{2}}$ for the modes from a localized source.

\section{References}

[1] A. A. Samarskii and A. N. Tikhonov, "Representation of the field in a waveguide as the sum of the TE and TM fields [O predstavlenii polya v volnovode v vide summy polej TE i TM]," Zhurnal tekhnicheskoy fiziki, vol. 18, no. 7, pp. 959-970, 1948, in Russian. 
[2] A. G. Sveshnikov, "A substantiation of a method for computing the propagation of electromagnetic oscillations in irregular waveguides," USSR Computational Mathematics and Mathematical Physics, vol. 3, no. 2, pp. 413-429, 1963. DOI: 10.1016/0041-5553(63)90027-2.

[3] V. V. Shevchenko, "Spectral decomposition in eigen- and associated functions of a nonselfadjoint problem of Sturm-Liouville type on the entire axis [O spektral'nom razlozhenii po sobstvennym i prisoedinennym funkciyam odnoj nesamosopryazhennoj zadachi tipa SHturma-Liuvillya na vsej osi]," Differ. Uravn., vol. 15, no. 11, pp. 2004-2020, 1979, in Russian.

[4] L. N. Deryugin, A. N. Marchuk, and V. E. Sotin, "Properties of planar asymmetrical dielectric waveguides on a substrate of dielectric [Svojstva planarnogo asimmetrichnogo dielektricheskogo volnovoda na podlozhke iz dielektrika]," Izv. Vyssh. Uchebn. Zaved., Ser. Radioelektron., vol. 10, no. 2, pp. 134-141, 1967, in Russian.

[5] A. G. Sveshnikov, "The incomplete Galerkin method [Nepolnyj metod Galerkina]," Dokl. Akad. Nauk SSSR, vol. 236, no. 5, pp. 1076-1079, 1977, in Russian.

[6] A. A. Bykov, A. G. Sveshnikov, and M. K. Trubetskov, "Reduced Galerkin's method application to calculations of eigenwaves in open waveguides [Primenenie nepolnogo metoda Galerkina dlya rascheta sobstvennyh voln otkrytyh volnovodov]," Matem. Mod., vol. 3, no. 7, pp. 111-123, 1991, in Russian.

[7] B. Z. Katsenelenbaum, Theory of Irregular Waveguides with Slowly Varying Parameters /Teoriya neregulyarnyh volnovodov s medlenno menyayushchimisya parametrami]. Moscow: Akad. Nauk SSSR, 1961, in Russian.

[8] V. V. Shevchenko, Continuous Transitions in Open Waveguides [Plavnye perekhody v otkrytyh volnovodah]. Moscow: Nauka, 1969, in Russian.

[9] L. A. Sevastianov and A. A. Egorov, "Theoretical analysis of the waveguide propagation of electromagnetic waves in dielectric smoothlyirregular integrated structures," Optics and Spectroscopy, vol. 105, no. 4, pp. 576-584, 2008. DOI: 10.1134/S0030400X08100123.

[10] A. A. Egorov and L. A. Sevastianov, "Structure of modes of a smoothly irregular integrated optical four-layer three-dimensional waveguide," Quantum Electronics, vol. 39, no. 6, pp. 566-574, 2009. DOI: 10.1070/ QE2009v039n06ABEH013966.

[11] A. A. Egorov, K. P. Lovetskiy, A. L. Sevastianov, and L. A. Sevastianov, "Simulation of guided modes (eigenmodes) and synthesis of a thin-film generalised waveguide Luneburg lens in the zero-order vector approximation," Quantum Electronics, vol. 40, no. 9, pp. 830-836, 2010. DOI: 10.1070/QE2010V040NO9ABEH014332.

[12] A. A. Egorov, A. L. Sevast'yanov, and L. A. Sevast'yanov, "Stable computer modeling of thin-film generalized waveguide Luneburg lens," Quantum Electronics, vol. 44, no. 2, pp. 167-173, 2014. DOI: 10.1070/ QE2014v044n02ABEH015303. 
[13] A. A. Egorov, A. L. Sevastyanov, E. A. Ayryan, and L. A. Sevastyanov, "Stable computer modeling of thin-film generalized waveguide Luneburg lens [Ustojchivoe komp'yuternoe modelirovanie tonkoplenochnoj obobshchennoj volnovodnoj linzy Lyunebergal," Matem. Mod., vol. 26, no. 11, pp. 37-44, 2014, in Russian.

[14] A. S. Il'inskii, V. V. Kravtsov, and A. G. Sveshnikov, Mathematical Models of Electrodynamics [Matematicheskie modeli elektrodinamiki]. Moscow: Vyssh. Shkola, 1991, in Russian.

[15] M. J. Adams, An Introduction to Optical Waveguides. New York: Wiley, 1981.

[16] T. Tamir, "Guided-Wave Optoelectronics," in Integrated Optics, T. Tamir, Ed. Berlin: Springer-Verlag, 1990.

[17] A. W. Snyder and J. D. Love, Optical Waveguide Theory. New York: Chapman and Hall, 1983.

[18] D. Markuze, Theory of Dielectric Optical Waveguides. New York: Academic Press, 1974.

[19] M. Barnoski, Introduction to Integrated Optics. New York: Plenum Press, 1974.

[20] A. I. Neishtadt, "On the accuracy of conservation of the adiabatic invariant," Journal of Applied Mathematics and Mechanics, vol. 45, no. 1, pp. 58-63, 1981. DOI: 10.1016/0021-8928(81)90010-1.

[21] I. V. Gorelyshev and A. I. Neishtadt, "On the adiabatic perturbation theory for systems with impacts," Journal of Applied Mathematics and Mechanics, vol. 70, no. 1, pp. 4-17, 2006. DOI: $10.1016 / \mathrm{j}$. jappmathmech.2006.03.015.

[22] V. M. Babich and V. S. Buldyrev, Asymptotic Methods in ShortWavelength Diffraction Theory, Alpha Science Series on Wave Phenomena. Harrow, UK: Alpha Science International, 2009.

[23] Y. A. Kravtsov and Y. I. Orlov, Geometrical Optics of Inhomogeneous Media. Berlin: Springer-Verlag, 1990.

[24] B. Z. Katsenelenbaum, "Irregular waveguides with slowly varying parameters [Neregulyarnye volnovody s medlenno menyayushchimisya parametrami]," Doklady Akademii Nauk SSSR, vol. 102, no. 4, p. 711, 1955, in Russian.

[25] B. Z. Katsenelenbaum, "A contribution to the general theory of nonregular wave guides [K obshchej teorii neregulyarnyh volnovodov]," Dokl. Akad. Nauk SSSR, vol. 116, no. 2, pp. 203-206, 1957, in Russian.

[26] A. I. Neishtadt, "Propagation of rays in smoothly irregular waveguides and perturbation theory of Hamiltonian systems [Rasprostranenie luchej $\mathrm{v}$ plavno neregulyarnyh volnovodah i teoriya vozmushchenij gamil'tonovyh sistem]," Izv. vuzov. Radiofizika, vol. 25, no. 2, pp. 218226, 1982, in Russian. 
[27] A. L. Sevastyanov, "Single-mode waveguide spread of light in a smooth irregular integral optical waveguide [Komp'yuternoe modelirovanie polej napravlyaemyh mod tonkoplenochnoj obobshchennoj volnovodnoj linzy Lyuneberga]," in Russian, Ph.D. dissertation, Peoples' Friendship University of Russia, Moscow, 2010.

[28] S. Solimeno, B. Crosignani, and P. DiPorto, Guiding, Diffraction, and Confinement of Optical Radiation. Orlando, FL: Academic Press, 1986.

[29] M. Kline and I. W. Kay, Electromagnenic Theory and Geometricak Optics. New York: Wiley (Inter-science), 1965.

[30] M. V. Fedoryuk, "A justification of the method of transverse sections for an acoustic wave guide with nonhomogeneous content," Mathematical Physics, vol. 13, no. 1, pp. 162-173, 1973. DoI: 10.1016/0041-5553(74) 90012-3.

\section{For citation:}

A. L. Sevastianov, Asymptotic method for constructing a model of adiabatic guided modes of smoothly irregular integrated optical waveguides, Discrete and Continuous Models and Applied Computational Science 28 (3) (2020) 252-273. DOI: $10.22363 / 2658-4670-2020-28-3-252-273$.

\section{Information about the authors:}

Sevastianov, Anton L. - Candidate of Physical and Mathematical Sciences, assistant professor of Department of Applied Probability and Informatics of Peoples' Friendship University of Russia (RUDN University) (e-mail: sevastianov-al@rudn.ru, phone: +7(495)9550927, ORCID: https://orcid.org/0000-0002-0280-485X, Scopus Author ID: 50462435500) 
DOI: $10.22363 / 2658-4670-2020-28-3-252-273$

\title{
Асимптотический метод построения модели адиабатических волноводных мод плавно-нерегулярных интегрально-оптических волноводов
}

\author{
А. Л. Севастьянов \\ Российский университет дружбы народов \\ ул. Миклухо-Маклал, д. 6, Москва, 117198, Россия
}

В работе рассмотрен класс плавно нерегулярных интегрально-оптических многослойных волноводов, свойства которых определяют характерные черты волноводного распространения в них монохроматического поляризованного света. Предложен асимптотический подход к описанию данного вида электромагнитного излучения, в результате которого решения системы уравнений Максвелла редуцируется к такому виду, который выражается через решения системы четырёх обыкновенных дифференциальных уравнений и двух алгебраических уравнений для шести компонент электромагнитного поля в нулевом приближении. Градиент фазового фронта адиабатической волноводной моды удовлетворяет уравнению эйконала относительно эффективного показателя преломления волновода относительно данной моды.

Многослойная структура волноводов позволяет произвести ещё один этап редукции системы уравнений модели к однородной системе линейных алгебраических уравнений, условие нетривиальной разрешимости которой задаёт связь градиента фазового фронта излучения с градиентами поверхностей раздела между тонкими однородными слоями.

В завершающей части работы сформулированы задачи (дифференциальная и алгебраическая) на собственные значения и собственные векторы для описания адиабатических волноводных мод. Приведена также формулировка задачи описания одномодового режима распространения адиабатических волноводных мод, подчёркивающая адиабатический характер описываемого приближенного решения уравнений Максвелла.

Ключевые слова: плавно нерегулярные интегрально-оптические многослойные волноводы, задачи на собственные значения и собственные векторы, одномодовый режим распространения адиабатических волноводных мод 